\title{
Platelet Indices and Their Kinetics Predict Mortality in Patients of Sepsis
}

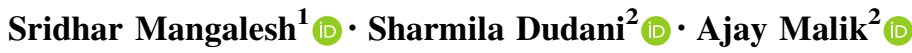

Received: 25 September 2020/Accepted: 9 February 2021/Published online: 24 March 2021

(C) Indian Society of Hematology and Blood Transfusion 2021

\begin{abstract}
Platelet indices are inexpensive, easily accessible parameters and potentially useful prognostic indicators in sepsis. In this study we explore the differences in platelet indices and their kinetics between sepsis survivors and nonsurvivors. A retrospective cohort-study of 97 cases of culture-positive sepsis at a tertiary-care center in North India. Demographics, clinical and laboratory parameters at admission were assessed. Platelet count (PLT), mean-platelet-volume (MPV), platelet-distribution-width (PDW) and plateletcrit (PCT) on admission, and third, fifth and last days of hospitalization were analyzed. Fractional change in platelet indices $\left(\Delta \mathrm{MPV}_{72 \mathrm{~h},}, \Delta \mathrm{PDW}_{72 \mathrm{~h}}, \Delta \mathrm{PCT}_{72 \mathrm{~h}}\right.$, and $\Delta \mathrm{PLT}_{72 \mathrm{~h}}$ ) by day-3 were calculated. Unpaired and paired t-tests were used to compare survivors with non-survivors, and to study the change in platelet indices with time. Logistic regression was used for multivariate analysis. ROC-curves and optimum cut-offs to predict mortality were obtained. There were 64 survivors. Non-survivors had significantly higher $\triangle \mathrm{MPV}_{72 \mathrm{~h}}, \Delta \mathrm{PDW}_{72 \mathrm{~h}}$, day-1 MPV and $\mathrm{PDW}$, and lower $\triangle \mathrm{PLT}_{72 \mathrm{~h}}$. MPV and PDW increased, and PLT decreased with time among non-survivors. Trends were reversed in survivors. Only MPV and PDW showed significant change by day-3. Both were independent predictors of mortality on multivariate analysis, alongside $\Delta \mathrm{MPV}_{72 \mathrm{~h}}$ and $\triangle \mathrm{PLT}_{72 \mathrm{~h}}$. On ROC analysis, MPV, PDW, $\Delta \mathrm{MPV}_{72 \mathrm{~h}}, \Delta \mathrm{PDW}_{72 \mathrm{~h}}$ and $\Delta \mathrm{PLT}_{72 \mathrm{~h}}$ effectively predicted mortality. Cut-off for MPV was 10.25 fL (sensitivity $=93.9 \%, \quad$ specificity $=60.9 \%)$, and PDW, $12.6 \%$
\end{abstract}

Sharmila Dudani

drsdudani@hotmail.com

Army College of Medical Sciences, Delhi, New Delhi, India

2 Department of Pathology, Army College of Medical Sciences, Delhi, New Delhi, India
$($ sensitivity $=84.8 \%$, specificity $=51.6 \%)$. A rise in MPV and a fall in PLT was associated with mortality in this study. MPV and PDW values at admission are effective predictors of mortality and may be used in conjunction with traditional parameters.

Keywords Mean platelet volume $\cdot$ Platelet count $\cdot$ Platelet function tests $\cdot$ Sepsis

\section{Introduction}

Sepsis is a constellation of various pathophysiological abnormalities that occurs due to a deleterious host response to infection, and eventually progresses to severe organ dysfunction, hypotension, septic shock, and death $[1,2]$. A major cause of morbidity and mortality in India and worldwide, it is a dreaded clinical entity met with continuous developments in diagnostics and therapeutics [3-5]. Sepsis and septic shock are driven by inflammatory responses to microbial components; activation of cytokine cascades, leukocyte induced damage, endothelial injury, peripheral vasodilation, and disseminated intravascular coagulation [6]. Lactate is a well-known marker of anaerobic metabolism and predicts sepsis mortality [7, 8]. Likewise, procalcitonin levels are upregulated in sepsis by microbial toxins and effectively predict mortality [9]. Both these parameters are among the most routinely used in clinical practice.

The utility of inflammatory markers in sepsis such as interleukin-6, pentraxin-3, and simpler hematological markers like immature neutrophil ratios and neutrophillymphocyte ratio (NLR) has been documented previously [10-12]. Pro-inflammatory cytokines in sepsis increase tissue factor production and induce a procoagulant state. 
Markers such as fibrinogen, prothrombin time, and antithrombin activity have been utilized to describe coagulopathy in sepsis $[13,14]$. Platelets have been described as 'critical key players' in sepsis, affecting outcomes through platelet-endothelial cell interactions and leading to sequential organ failure [15]. Platelet indices include the platelet distribution width (PDW), which increases during accelerated platelet turnover; the mean platelet volume (MPV), indicative of platelet size; and plateletcrit (PCT), a measure of total platelet mass. These are inexpensive, easily accessible parameters available on a complete hemogram, but remain underutilized [16].

Although platelet indices have been described for their prognostic utility in sepsis and septic shock, there is a relative scarcity of literature on the same. Findings from previous studies on MPV have been largely inconsistent. Some studies show higher MPV levels in non-survivors, whereas others establish the reverse [17-20]. In this study, we analyze the kinetics of platelet indices in patients with sepsis. We also compare platelet indices at the time of admission between survivors and non-survivors and determine appropriate cut-offs for predicting mortality.

\section{Materials and Methods}

This retrospective cohort study was conducted at a tertiarycare center in North India. Patient records of 97 cases of sepsis between January and August 2020 were analyzed.

Sepsis was defined as per the Third International Consensus Definitions for Sepsis and Septic Shock (Sepsis-3) [1]. Records of adult patients admitted to the intensive care unit (ICU) who had blood-culture confirmed sepsis were evaluated. Recovered patients discharged from the ICU were placed in the survivor cohort, and those who succumbed, in the non-survivor cohort. Pregnant women, lactating mothers, patients who expired within five days of admission, patients who received platelet transfusions or fresh frozen plasma, and patients for whom investigations for all platelet indices were unavailable were excluded from the analysis. Patients with hematological malignancies, hypersplenism, immune thrombocytopenic purpura, reactive thrombocytosis, or essential thrombocythemia were also excluded.

Demographic information collected included patient age, sex, and the presence of comorbid conditions. The primary site of infection was noted. Complete hemogram parameters obtained on admission and the third, fifth, and last day of ICU stay were studied. These consisted of red cell counts (RBC), hemoglobin ( $\mathrm{Hb})$, total and differential leukocyte counts (TLC and DLC), NLR, and platelet indices, including platelet count (PLT), MPV, PDW, and PCT. Blood was collected in appropriate vacutainers using a closed draw vacuum system. Samples were not subjected to storage, and investigations were reported within $1.5 \mathrm{~h}$. Cell counts were estimated using the Sysmex ${ }^{\circledR}$ KX-21 (Sysmex Corp, Japan) cell counter. All samples were subjected to a concomitant smear examination as well. Clinical parameters on admission, including vitals, routine investigations including renal and liver function tests, arterial blood gases, lactate levels, the need for ventilator support, an associated surgical intervention, and microbiological reports were also analyzed. Procalcitonin level on admission was estimated using VIDAS $^{\circledR}$ B.R.A.H.M.S PCT (Biomérieux, France).

The normal reference intervals for the platelet parameters were; PLT $\left(150-400 \times 10^{3} / \mathrm{uL}\right)$, MPV (6.5-12.0 fL), PDW (9.0-17.0\%) and PCT $(0.15-0.38 \%)$. The fractional change in the MPV from the 1st day (on admission) to the 3rd day was calculated as $\Delta \mathrm{MPV}_{72 \mathrm{~h}}=(\mathrm{MPV}$ on day-3MPV on day-1) $\div$ (MPV on day-1). A similar treatment of the other platelet indices was used to obtain $\Delta \mathrm{PDW}_{72 \mathrm{~h}}$, $\Delta \mathrm{PCT}_{72 \mathrm{~h}}$, and $\Delta \mathrm{PLT}_{72 \mathrm{~h}}$.

Statistical analysis was performed using SPSS 21.0 for Windows (Armonk, NY: IBM Corp). Continuous variables are expressed as mean \pm standard deviation (SD) or medians with interquartile range (IQR), and categorical variables as percentages. Normality assumptions were tested using the Shapiro-Wilk test, and the homogeneity of variances assumption was tested using Levene's test. We used a paired-samples t-test to assess the change in platelet indices with time compared to the values on admission, and an independent-samples t-test to compare parameters between the survivor and non-survivor cohorts. Non-parametric variants of tests were used where appropriate. Association between categorical variables was assessed using the Chi-square test or Fisher's exact test. Binary logistic regression was used for multivariate analysis of platelet indices and other variables for predicting mortality. Receiver operator characteristic (ROC) curves were plotted to study the utility of different parameters as mortality predictors. Optimal cut-offs with sensitivity, specificity, and positive and negative predictive values (PPV and NPV) are reported. All $p$ values $<0.05$ were considered statistically significant.

\section{Results}

A total of 97 patients, comprising 64 (65.98\%) survivors and $33(34.02 \%)$ non-survivors, were studied. Baseline parameters at the time of admission were comparable between survivors and non-survivors (Table 1). Comorbid conditions observed included type-2 diabetes mellitus, hypertension, coronary artery disease (CAD), or a combination of these, present in 48 patients. Hypertension was 
Table 1 Baseline parameters of survivor and non-survivor groups on admission

\begin{tabular}{|c|c|c|c|}
\hline \multirow{2}{*}{$\begin{array}{l}\text { Parameter } \\
\text { Age (years) }\end{array}$} & \multicolumn{3}{|c|}{ Mean \pm SD, Median $(\mathrm{IQR})$ or $\mathrm{n}[\%]$} \\
\hline & $67.06 \pm 9.98$ & $68.24 \pm 8.18$ & 0.560 \\
\hline Sex & & & 0.449 \\
\hline Male & $40[62.5 \%]$ & $18[54.5 \%]$ & \\
\hline Female & $24[37.5 \%]$ & $15[45.5 \%]$ & \\
\hline Temperature $\left({ }^{\mathrm{o}} \mathrm{F}\right)$ & $100.5(1.0)$ & $101.0(1.5)$ & 0.098 \\
\hline $\operatorname{HR}\left(\min ^{-1}\right)$ & $110.28 \pm 9.35$ & $113.73 \pm 9.86$ & 0.095 \\
\hline MAP (mmHg) & $74.00(9.17)$ & 72.67 (12.67) & 0.366 \\
\hline $\mathrm{pH}$ & $7.357 \pm 0.043$ & $7.346 \pm 0.046$ & 0.261 \\
\hline $\mathrm{SaO} 2(\%)$ & $92.72 \pm 3.27$ & $91.94 \pm 3.31$ & 0.271 \\
\hline Bicarbonate (mEq/L) & $24.02 \pm 2.10$ & $23.36 \pm 1.39$ & 0.071 \\
\hline $\mathrm{RBC}\left(\times 10^{6} / \mathrm{uL}\right)$ & $4.69 \pm 0.74$ & $4.56 \pm 0.81$ & 0.442 \\
\hline $\mathrm{Hb}(\mathrm{g} / \mathrm{dL})$ & $12.35(1.50)$ & $12.00(1.60)$ & 0.154 \\
\hline TLC $\left(\times 10^{3} / \mathrm{uL}\right)$ & $13.50(4.02)$ & $14.6(2.15)$ & 0.155 \\
\hline $\mathrm{PT}(\mathrm{sec})$ & $13.0(1.0)$ & $14.0(2.0)$ & 0.313 \\
\hline INR & $0.90(0.1)$ & $1.00(0.2)$ & 0.313 \\
\hline Total Bilirubin (mg/dL) & $0.83(0.36)$ & $0.80(0.34)$ & 0.684 \\
\hline Direct Bilirubin (mg/dL) & $0.12(0.11)$ & $0.15(0.10)$ & 0.265 \\
\hline $\mathrm{ALT}(\mathrm{U} / \mathrm{L})$ & $40.0(13.0)$ & $38.0(13.0)$ & 0.930 \\
\hline AST (U/L) & $39.0(10.0)$ & $41.0(32.0)$ & 0.269 \\
\hline ALP (U/L) & $70.0(17.0)$ & $74.0(14.0)$ & 0.117 \\
\hline Protein $(\mathrm{g} / \mathrm{dL})$ & $6.053 \pm 0.943$ & $5.945 \pm 1.140$ & 0.621 \\
\hline Albumin (g/dL) & $3.212 \pm 0.947$ & $3.167 \pm 0.927$ & 0.820 \\
\hline Globulin (g/dL) & $2.80(0.30)$ & $3.0(0.60)$ & 0.155 \\
\hline Urea $(\mathrm{mg} / \mathrm{dL})$ & $30.50(9.0)$ & $29.00(10.0)$ & 0.164 \\
\hline Creatinine (mg/dL) & $0.989 \pm 0.192$ & $0.991 \pm 0.428$ & 0.978 \\
\hline Microbiology & & & 0.343 \\
\hline Gram Negative & $41[64.1 \%]$ & $16[48.5 \%]$ & \\
\hline Gram Positive & $20[31.2 \%]$ & $15[45.5 \%]$ & \\
\hline Fungi & $3[4.7 \%]$ & $2[6.1 \%]$ & \\
\hline Origin of Infection & & & 0.609 \\
\hline Intra-Abdominal & $30[46.9 \%]$ & $11[33.3 \%]$ & \\
\hline Pulmonary & $27[42.2 \%]$ & $17[51.5 \%]$ & \\
\hline Urinary Tract & $3[4.7 \%]$ & $2[6.1 \%]$ & \\
\hline CNS & $4[6.2 \%]$ & $3[9.1 \%]$ & \\
\hline Comorbidities $(\geq 1)$ & $29[45.3 \%]$ & $19[57.6 \%]$ & 0.252 \\
\hline Surgical Intervention & $5[7.8 \%]$ & $4[12.1 \%]$ & 0.484 \\
\hline Ventilator Support & 14 [21.9\%] & $6[18.2 \%]$ & 0.670 \\
\hline
\end{tabular}

*Unpaired t-test/Mann Whitney U test/Chi Square test/Fisher's Exact test present in 14, diabetes in 12, and CAD in 6 patients. Both diabetes and hypertension were present in 6 , diabetes and CAD in 3, and hypertension and CAD in 3 patients. All three conditions were present in 4 patients.

Procalcitonin, lactate, MPV, and PDW values were significantly higher among non-survivors at admission. $\Delta \mathrm{MPV}_{72 \mathrm{~h}}, \Delta \mathrm{PDW}_{72 \mathrm{~h}}$, and $\triangle \mathrm{PCT}_{72 \mathrm{~h}}$ values were negative in survivors and positive in non-survivors, and conversely, $\Delta \mathrm{PLT}_{72 \mathrm{~h}}$ was positive in survivors and negative in non- survivors. Only $\Delta \mathrm{MPV}_{72 \mathrm{~h}}, \Delta \mathrm{PDW}_{72 \mathrm{~h}}$, and $\Delta \mathrm{PLT}_{72 \mathrm{~h}}$ showed a statistically significant difference between the two cohorts. No differences were observed for PLT, PCT, and NLR values at admission (Table 2).

Platelet indices on the third, fifth, and last day of hospitalization were compared with baseline values (day-1) using a repeated measures design (Table 3 ). The time trends of these parameters for the survivor and non-survivor cohorts are represented graphically in (Fig. 1). 
Table 2 Platelet indices, NLR, lactate, procalcitonin levels, $\Delta \mathrm{MPV}_{72 \mathrm{~h}}, \Delta \mathrm{PDW}_{72 \mathrm{~h}}, \Delta \mathrm{PCT}_{72 \mathrm{~h}}$, and $\triangle \mathrm{PLT}_{72 \mathrm{~h}}$ compared amongst the survivor and nonsurvivor groups

\begin{tabular}{|c|c|c|c|}
\hline Parameter & $\begin{array}{l}\text { Survivors } \mathrm{n}=64 \\
\text { Mean } \pm \mathrm{SD} \text { or } \mathrm{M}\end{array}$ & $\begin{array}{l}\text { Non-Survivors } n=33 \\
\text { R) }\end{array}$ & $P$ Value \\
\hline $\operatorname{PLT}\left(\times 10^{3} / \mathrm{uL}\right)$ & $175.69 \pm 43.07$ & $158.39 \pm 45.83$ & 0.070 \\
\hline PCT $(\%)$ & $18.10 \pm 3.98$ & $17.38 \pm 3.48$ & 0.379 \\
\hline MPV (fL) & $10.06 \pm 1.48$ & $11.91 \pm 1.31$ & $<0.0001$ \\
\hline PDW (\%) & $12.95 \pm 3.69$ & $14.3 \pm 2.10$ & 0.024 \\
\hline NLR & $9.66 \pm 0.87$ & $10.1 \pm 1.71$ & 0.167 \\
\hline Day 5 NLR & $9.29 \pm 1.49$ & $11.10 \pm 1.50$ & 0.0001 \\
\hline Lactate $(\mathrm{mmol} / \mathrm{L})$ & $1.60(1.30)$ & $2.70(0.85)$ & $<0.0001$ \\
\hline Procalcitonin (ng/mL) & $0.22(0.54)$ & $1.67(1.31)$ & $<0.0001$ \\
\hline$\Delta \mathrm{MPV}_{72 \mathrm{~h}}$ & $0.027 \pm 0.096$ & $0.031 \pm 0.073$ & 0.003 \\
\hline$\Delta \mathrm{PDW}_{72 \mathrm{~h}}$ & $0.012 \pm 0.089$ & $0.026 \pm 0.075$ & 0.036 \\
\hline$\Delta \mathrm{PCT}_{72 \mathrm{~h}}$ & $0.019(0.0780)$ & $0.005(0.0720)$ & 0.270 \\
\hline$\Delta \mathrm{PLT}_{72 \mathrm{~h}}$ & $0.006 \pm 0.047$ & $0.029 \pm 0.070$ & 0.013 \\
\hline
\end{tabular}

Bold indicates statistically significant with $P<0.05$

Table 3 Kinetics of Platelet Indices and NLR amongst survivors and non-survivors. Values on Day 3, Day 5 and the Last Day are compared with the baseline (Day 1) using a paired samples design

\begin{tabular}{|c|c|c|c|c|c|c|c|}
\hline \multicolumn{8}{|l|}{ Survivors } \\
\hline Parameter & Day 1 & Day 3 & $P$ Value & Day 5 & $P$ Value & Last Day ${ }^{a}$ & $P$ Value \\
\hline PLT $\left(\times 10^{3} / \mathrm{uL}\right)$ & $175.7 \pm 43.1$ & $176.8 \pm 43.4$ & 0.245 & $178.5 \pm 43.0$ & 0.008 & $180.3 \pm 43.1$ & 0.001 \\
\hline PCT $(\%)$ & $18.10 \pm 3.98$ & $17.89 \pm 4.02$ & 0.112 & $18.31 \pm 3.99$ & 0.125 & $19.01 \pm 4.29$ & 0.003 \\
\hline MPV (fL) & $10.06 \pm 1.48$ & $9.80 \pm 1.81$ & 0.034 & $9.73 \pm 1.61$ & 0.023 & $9.50 \pm 1.72$ & 0.0008 \\
\hline PDW (\%) & $12.95 \pm 3.69$ & $12.87 \pm 3.95$ & 0.545 & $12.10 \pm 2.09$ & 0.016 & $12.02 \pm 2.17$ & 0.010 \\
\hline NLR & $9.66 \pm 0.87$ & $9.81 \pm 1.43$ & 0.238 & $9.29 \pm 1.49$ & 0.014 & $8.95 \pm 2.70$ & 0.028 \\
\hline \multicolumn{8}{|l|}{ Non-Survivors } \\
\hline Parameter & Day 1 & Day 3 & $P$ Value & Day 5 & $P$ Value & Last Day ${ }^{\mathrm{b}}$ & $P$ Value \\
\hline PLT $\left(\times 10^{3} / \mathrm{uL}\right)$ & $158.4 \pm 45.8$ & $155.3 \pm 50.1$ & 0.089 & $151.3 \pm 50.6$ & 0.0003 & $140.9 \pm 52.0$ & $<0.0001$ \\
\hline PCT $(\%)$ & $17.38 \pm 3.48$ & $17.32 \pm 3.67$ & 0.724 & $17.14 \pm 3.88$ & 0.337 & $17.06 \pm 3.91$ & 0.271 \\
\hline MPV (fL) & $11.91 \pm 1.31$ & $12.24 \pm 1.14$ & 0.033 & $12.44 \pm 0.94$ & 0.005 & $13.19 \pm 1.46$ & $<0.0001$ \\
\hline PDW (\%) & $14.30 \pm 2.10$ & $14.66 \pm 2.26$ & 0.045 & $14.91 \pm 2.29$ & 0.002 & $15.44 \pm 2.31$ & $<0.0001$ \\
\hline NLR & $10.11 \pm 1.71$ & $10.50 \pm 1.96$ & 0.059 & $11.10 \pm 1.49$ & 0.0001 & $11.18 \pm 1.51$ & 0.00026 \\
\hline
\end{tabular}

Bold indicates statistically significant with $P<0.05$

${ }^{a}$ Last Day $=$ date of discharge for survivors

${ }^{\mathrm{b}}$ Last Day $=$ date of demise for non-survivors

Compared to baseline, MPV was significantly higher among non-survivors and lower among survivors on all the three days studied. Other platelet indices significantly deviated from baseline values only on the fifth or last days. Overall, PLT and PCT increased among survivors and decreased among non-survivors during hospitalization. PDW and NLR increased among non-survivors and decreased among survivors.

The range of thrombocytopenia (in $\times 10^{3} / \mathrm{uL}$ ) seen on day-1 was 158.39 [142.14-174.64] (Minimum:82, Maximum:246) in non-survivors and 175.69 [164.93-186.45] (Minimum:70, Maximum:271) in survivors. On day-3, it was 155.33 [137.55-173.12] (Minimum:65, Maximum:256) in non-survivors and 176.77 [165.92-187.61] (Minimum:63, Maximum:265) in survivors. On day-5, it was 151.27 [133.35-169.20] (Minimum:60, Maximum:252) in non-survivors, and 178.52 [167.76-189.27] (Minimum:65, Maximum:267) in survivors. On the last day, it was 140.94 [122.51-159.37] (Minimum:54, Maximum:244) in non-survivors and 180.34 [169.58-191.10] (Minimum:67, Maximum:272) in survivors.

Binary logistic regression was used to assess platelet indices, patient age, and comorbid conditions as mortality predictors (Table 4). On multivariate analysis, MPV and 

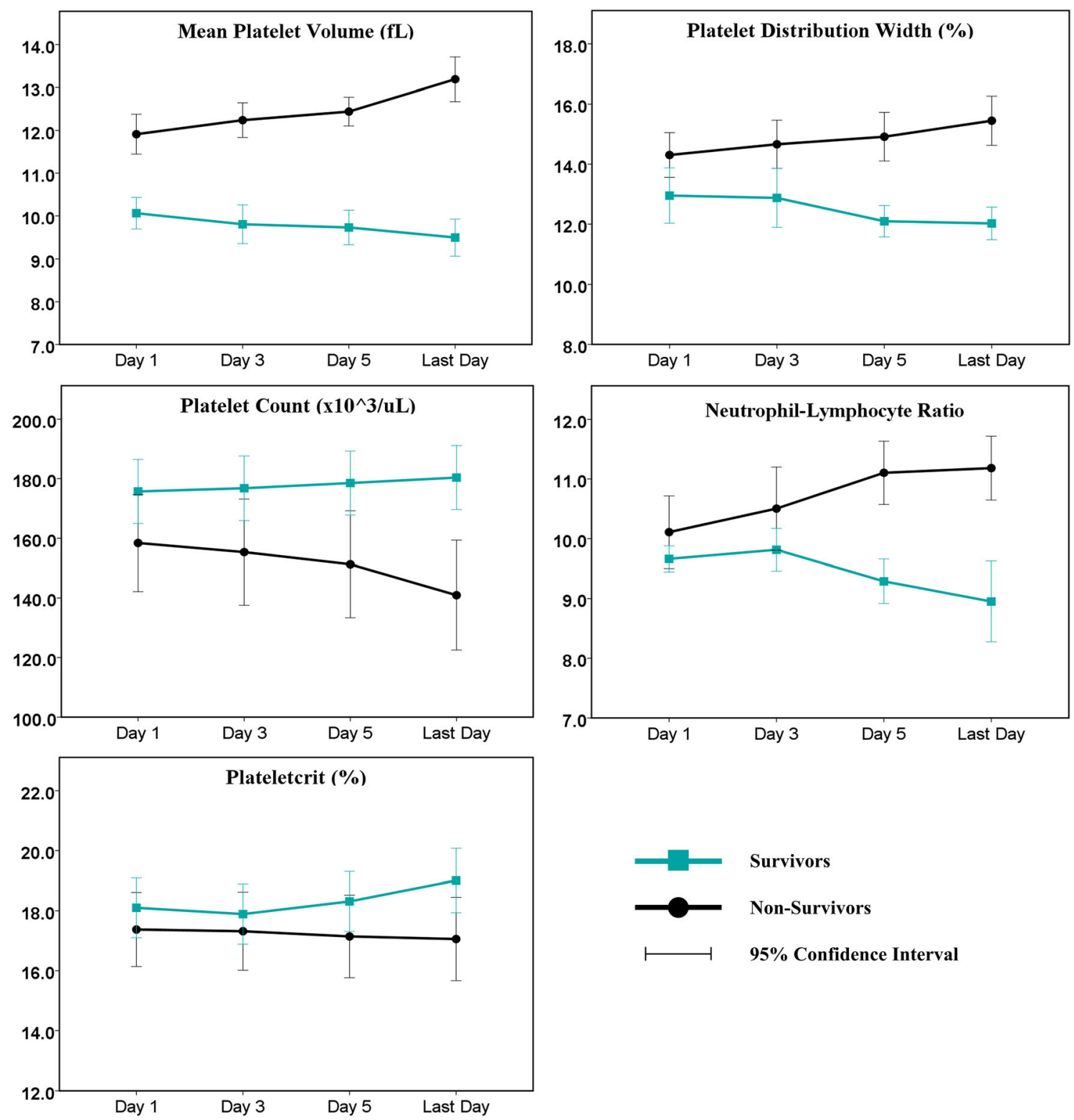

Fig. 1 Change in Platelet indices and NLR with time in the survivor and non-survivor groups

PDW were significant predictors of mortality. Among platelet kinetics, $\triangle \mathrm{MPV}_{72 \mathrm{~h}}$ and $\triangle \mathrm{PLT}_{72 \mathrm{~h}}$ were independent predictors of mortality on adjusting for patient age and comorbid conditions.

ROC curves for procalcitonin, lactate, MPV, PDW, $\Delta \mathrm{MPV}_{72 \mathrm{~h}}, \Delta \mathrm{PDW}_{72 \mathrm{~h}}$, and $\Delta \mathrm{PLT}_{72 \mathrm{~h}}$ were constructed (Fig. 2). Procalcitonin and lactate had the highest AUCs (0.909 and 0.877, respectively). MPV was the third-best mortality predictor, with an AUC $=0.825$ and an optimum cut-off of $10.25 \mathrm{fL}$ with a sensitivity of $93.9 \%$ and specificity of $60.9 \%$. Other parameters showed moderate AUC values. The results are summarized in (Fig. 2).

\section{Discussion}

Platelet indices vary significantly between sepsis survivors and non-survivors on admission. A distinct pattern of change in these parameters was observed during the clinical course of sepsis, which may have important clinical implications. Most notably, the MPV was significantly higher amongst non-survivors on admission, consistently increased with time in this group, and decreased among survivors. A higher MPV is indicative of increased platelet turnover by the bone marrow in response to stress, and smaller platelets are suggestive of platelet maturity. Younger, larger platelets are also functionally more active. With increased platelet activation in septic patients, 
Table 4 Binary logistic regression with univariate (unadjusted) and multivariate (adjusted) odds ratios for platelet parameters and comorbid conditions as mortality predictors

\begin{tabular}{|c|c|c|c|c|}
\hline Predictor & Unadjusted Odds & $P$ Value & Adjusted Odds & $P$ Value \\
\hline \multicolumn{5}{|c|}{ Model 1: Platelet Indices ${ }^{a}$} \\
\hline Patient Age & $1.014(0.969-1.061)$ & 0.556 & $0.993(0.934-1.056)$ & 0.816 \\
\hline $\operatorname{PLT}\left(\times 10^{3} / \mathrm{uL}\right)$ & $0.991(0.981-1.001)$ & 0.073 & $0.994(0.979-1.008)$ & 0.401 \\
\hline MPV (fL) & $2.441(1.655-3.600)$ & $0.0001 * *$ & $2.603(1.694-3.999)$ & $0.0001 * *$ \\
\hline PDW (\%) & $1.144(0.995-1.316)$ & 0.059 & $1.252(1.023-1.531)$ & $0.029 *$ \\
\hline PCT $(\%)$ & $0.951(0.850-1.063)$ & 0.375 & $1.001(0.835-1.199)$ & 0.992 \\
\hline \multicolumn{5}{|l|}{ Diabetes } \\
\hline No & Reference & - & Reference & - \\
\hline Yes & $1.786(0.701-4.551)$ & 0.224 & $1.442(0.422-4.923)$ & 0.559 \\
\hline \multicolumn{5}{|l|}{ Hypertension } \\
\hline No & Reference & - & Reference & - \\
\hline Yes & $1.867(0.748-4.661)$ & 0.181 & $1.111(0.292-4.221)$ & 0.878 \\
\hline \multicolumn{5}{|l|}{ CAD } \\
\hline No & Reference & - & Reference & - \\
\hline Yes & $2.240(0.755-6.646)$ & 0.146 & $3.330(0.753-14.727)$ & 0.113 \\
\hline Intercept $(\beta)$ & - & - & -13.095 & - \\
\hline \multicolumn{5}{|c|}{ Model 2: Platelet Kinetics ${ }^{b}$} \\
\hline Patient Age & $1.014(0.969-1.061)$ & 0.556 & $0.989(0.934-1.048)$ & 0.716 \\
\hline$\Delta \mathrm{PLT}_{72 \mathrm{~h}}(\%)$ & $0.895(0.825-0.971)$ & $0.007 * *$ & $0.906(0.828-0.991)$ & $0.030 *$ \\
\hline$\Delta \mathrm{MPV}_{72 \mathrm{~h}}(\%)$ & $1.078(1.023-1.136)$ & $0.005 * *$ & $1.077(1.019-1.138)$ & $0.009 * *$ \\
\hline$\Delta \mathrm{PDW}_{72 \mathrm{~h}}(\%)$ & $1.057(1.002-1.115)$ & $0.042^{*}$ & $1.061(0.994-1.132)$ & 0.074 \\
\hline$\Delta \mathrm{PCT}_{72 \mathrm{~h}}(\%)$ & $1.017(0.949-1.090)$ & 0.633 & $1.012(0.935-1.095)$ & 0.765 \\
\hline \multicolumn{5}{|l|}{ Diabetes } \\
\hline No & Reference & - & Reference & - \\
\hline Yes & $1.786(0.701-4.551)$ & 0.224 & $1.414(0.438-4.566)$ & 0.563 \\
\hline \multicolumn{5}{|l|}{ Hypertension } \\
\hline No & Reference & - & Reference & - \\
\hline Yes & $1.867(0.748-4.661)$ & 0.181 & $1.363(0.440-4.223)$ & 0.591 \\
\hline \multicolumn{5}{|l|}{$C A D$} \\
\hline No & Reference & - & Reference & - \\
\hline Yes & $2.240(0.755-6.646)$ & 0.146 & $2.161(0.554-8.422)$ & 0.267 \\
\hline Intercept $(\beta)$ & - & - & 0.399 & - \\
\hline
\end{tabular}

In brackets: 95\% Confidence Intervals of odds ratios.

*significant at $P<0.05$;

**significant at $P<0.01$.

${ }^{\mathrm{a} C o x}-$ Snell $\mathrm{R}^{2}=0.345$; Nagelkerke $\mathrm{R}^{2}=477$;

${ }^{\mathrm{b}}$ Cox-Snell $\mathrm{R}^{2}=0.292$; Nagelkerke $\mathrm{R}^{2}=211$. platelets with augmented thrombotic potential show spontaneous aggregation in the circulation [21-23].

Previous studies have found elevated MPV levels in sepsis non-survivors, which return to normal after one week of effective antibiotic therapy [17, 18, 24]. Presumably, a high MPV is an indicator of an active and invasive systemic infection. Conflicting evidence exists regarding MPV in sepsis, with some studies reporting a lower level of MPV in non-survivors [19, 20]. Others propose more complex patterns of MPV changes-an initial rise of MPV in septicemia and a subsequent fall in sustained bacterial infections [25]. Our study demonstrates that a high and rising MPV is associated with severity and poor outcomes, and is valuable for dynamic prognostication of sepsis.

PDW changes paralleled MPV in this study, albeit to a moderate degree. The PDW values at admission were significantly higher among non-survivors and increased further with time. A high PDW indicates platelet heterogeneity, resulting from platelet swelling in the circulation and platelet immaturity. This finding is in agreement with other studies [17, 26, 27]. Both PLT and PCT did not vary significantly between survivors and non-survivors on 


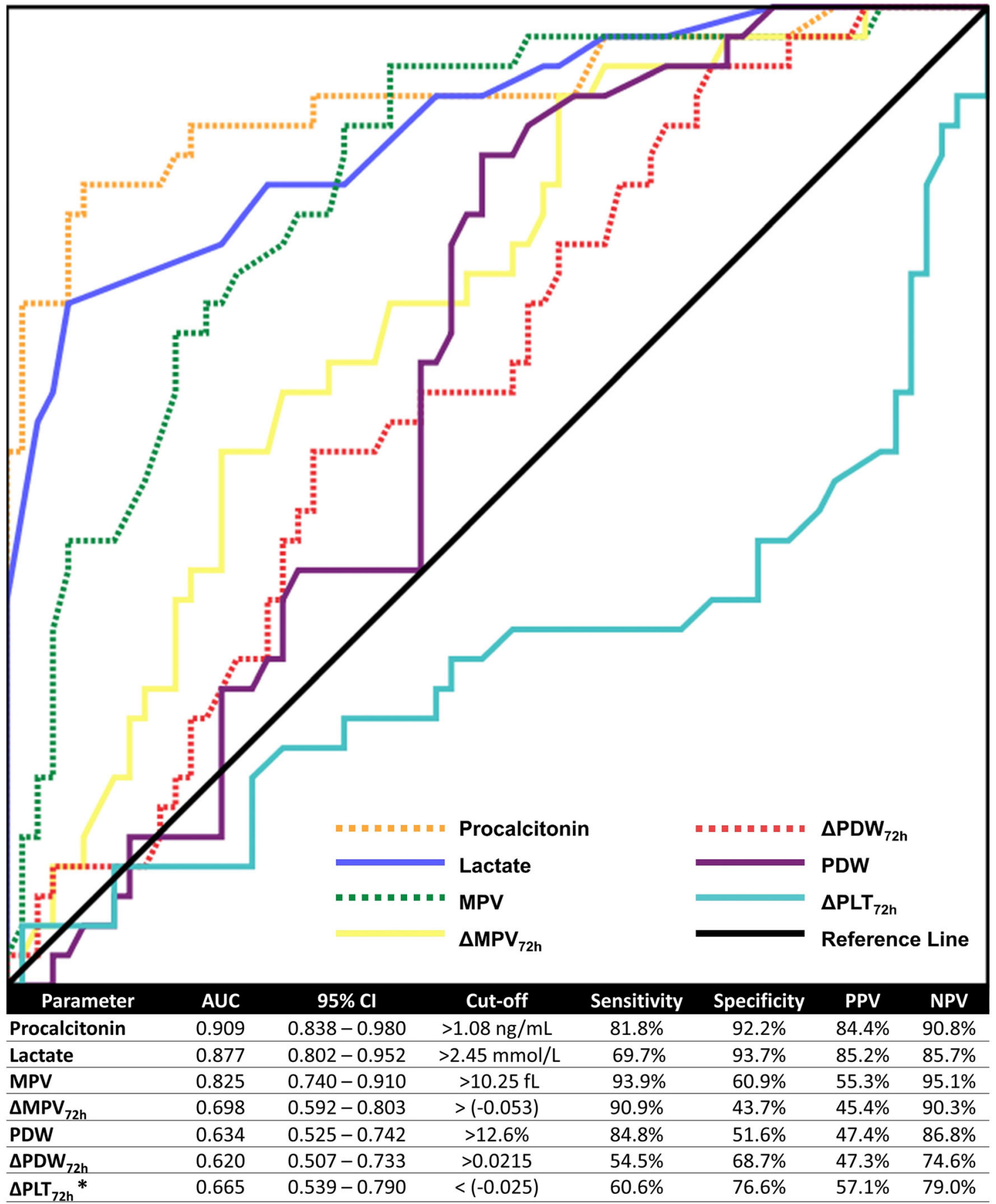

Fig. 2 ROC curves for platelet indices, lactate and procalcitonin levels to predict mortality. ${ }^{*} \mathrm{~A}$ smaller value of $\Delta \mathrm{PLT}_{72 \mathrm{~h}}$ favors mortality, unlike the other variables. The test direction was reversed and the AUC and cut-offs were calculated accordingly

admission but showed predictable time trends. PLT counts decreased over time in non-survivors, and both PLT and PCT values improved over time among survivors. Thrombocytopenia due to diminished production or increased turnover is a well-recognized consequence of sepsis, leading to hemorrhagic complications that arise from the consumption of platelets and coagulation factors. Persistent thrombocytopenia is associated with worse outcomes, and a single initial platelet count does not discriminate survivors from non-survivors [28, 29]. NLR at admission did not vary significantly between survivors and non-survivors but differed significantly on Day-5 and increased among non-survivors with disease progression. The NLR is a simple marker of systemic inflammation and stress, and a previous study has similarly described Day-5 NLR as a late phase mortality indicator of sepsis, but Day- 
1 NLR was not significant [12]. Early phase predictors of mortality have greater clinical utility. These findings suggest that NLR does not change significantly at the onset of sepsis, showing detectable changes only later in the disease course.

Lactate and procalcitonin values varied significantly between the two cohorts in our study. Lactate level rises following tissue hypoperfusion and anaerobic metabolism and is a fundamental danger sign in sepsis [7, 8]. Procalcitonin is a useful marker for both risk-stratification and prognostication in sepsis, which helps in guiding antibiotic use [9, 30]. Interestingly, we found that procalcitonin had a slightly higher AUC than lactate in this study. This is perhaps explained by the inclusion of only blood-culture positive patients with sepsis in our study [31].

We constructed ROC curves to compare platelet indices, procalcitonin and lactate in predicting mortality. After lactate and procalcitonin (AUC $=0.877$ and 0.909), MPV (AUC $=0.825$ ) was the next best parameter with an optimum cut-off of $10.25 \mathrm{fL}$. The sensitivity was $93.9 \%$, which was higher than that of both procalcitonin $(81.8 \%)$ and lactate $(69.7 \%)$. A previous study on a cohort of septic shock patients reported a cut-off of $10.5 \mathrm{fL}$ for MPV [17]. Another reports a cut-off of $8.0 \mathrm{fL}$ for the diagnosis of sepsis versus non-septic controls [19]. PDW had a moderate AUC $=0.634$, with an optimum cut-off of $12.6 \%$, and a sensitivity of $84.8 \%$. However, both MPV and PDW had relatively poor specificities and PPV compared to lactate and procalcitonin. Interestingly, most patients in our study had MPV and PDW values in the normal range on admission, and the average values of MPV and PDW among both survivors and non-survivors were within the physiological reference ranges. The same is true for the cut-off values of MPV and PDW discussed earlier. Despite no definitive deviation beyond the critical point, even nuanced variations in these parameters predicted mortality. It may hence be prudent to consider the time-trends of platelet indices for monitoring patients of sepsis, as conventional reference ranges may be unsuitable.

Previous studies have emphasized the significance of the kinetics of platelet indices, especially over the first $72 \mathrm{~h}$ following admission [32, 33]. We calculated $\Delta \mathrm{MPV}_{72 \mathrm{~h}}$, $\Delta \mathrm{PDW}_{72 \mathrm{~h}}, \Delta \mathrm{PCT}_{72 \mathrm{~h}}$, and $\triangle \mathrm{PLT}_{72 \mathrm{~h}}$, which indicated the fractional change in platelet indices over the first three days of admission. $\triangle \mathrm{MPV}_{72 \mathrm{~h}}, \Delta \mathrm{PDW}_{72 \mathrm{~h}}$, and $\triangle \mathrm{PLT}_{72 \mathrm{~h}}$ varied significantly between survivors and non-survivors, and all three had moderate AUC values on ROC analysis. The optimum cut-off for $\triangle \mathrm{PDW}_{72 \mathrm{~h}}$ was 0.0215 , which implies that an increase in PDW by more than $2.15 \%$ over the first $72 \mathrm{~h}$ favored mortality. The cut-offs for $\Delta \mathrm{PLT}_{72 \mathrm{~h}}$ and $\triangle \mathrm{MPV}_{72 \mathrm{~h}}$ had a negative sign and were 0.025 and 0.053 , respectively. A fall in the PLT greater than $2.5 \%$ in the first $72 \mathrm{~h}$ was associated with an increased risk of mortality.
Similarly, an MPV value that did not decrease by at least $5.3 \%$ over the first $72 \mathrm{~h}$ increased the risk of mortality. In conjunction with early markers, monitoring the change in platelet indices over time, particularly over the first few days of admission, provides unique insight into the prognosis of sepsis.

Platelet indices are easily accessible hematology investigations that are routinely available in all complete hemogram reports. They are inexpensive and require small sample volumes and time for estimation, making them ideal for low-resource settings. Changes in platelet indices over time are easily estimated using simple arithmetic. The use of sophisticated assays may not always be feasible, creating undue strain on the healthcare system, especially when repeat serial measurements are required. Simple, cost-effective hematological markers, therefore, assume great significance.

\section{Limitations}

Sepsis mortality and platelet indices are closely associated, but this study does not fully elucidate any underlying cause-effect relationships. Individual therapeutic decisions for each patient have implications on outcomes that could not be investigated. As this was a retrospective study, several factors could be out of the authors' control and subject to inconsistencies. Long-term survival outcomes and 28-day mortality data of patients in this study could not be analyzed. A prospective study design enables more complete and meticulous data collection and management. Prospective studies on larger patient populations are needed to adjust for all possible confounding variables more robustly.

\section{Conclusion}

Platelet indices are inexpensive, easily accessible parameters that may be useful prognostic indicators in sepsis. A rise in MPV, PDW, and a fall in PLT was associated with mortality in this study. An MPV of $>10.25 \mathrm{fL}$ and PDW $>12.6 \%$ at admission were effective predictors of mortality.

Funding This research did not receive any specific grant from funding agencies in the public, commercial, or not-for-profit sectors.

\section{Compliance with Ethical Standards}

Conflict of interest The authors declare that they have no conflict of interest. 
Ethics Approval This retrospective study was conducted according to the principles of the Declaration of Helsinki. The study was approved by the scientific review committee and ethics committee of the institution (No. 1156/IEC/07/2020/18, dated $31^{\text {st }}$ July 2020).

Availability of Data Raw data used in the analysis will be made available on request.

Consent to Participate and for Publication In view of the retrospective nature of the study, patient consent was not needed.

\section{References}

1. Singer M, Deutschman CS, Seymour CW, Shankar-Hari M, Annane D, Bauer $\mathrm{M}$ et al (2016) The third international consensus definitions for sepsis and septic shock (Sepsis-3). JAMA 315:801-810

2. Dellinger RP, Levy MM, Rhodes A, Annane D, Gerlach H, Opal SM et al (2013) Surviving sepsis campaign guidelines committee including the pediatric subgroup. Surviving sepsis campaign: international guidelines for management of severe sepsis and septic shock: 2012. Crit Care Med 41:580-637

3. Chatterjee S, Bhattacharya M, Todi SK (2017) Epidemiology of adult-population sepsis in India: a single center 5 year experience. Indian J Crit Care Med 21:573-577

4. Linde-Zwirble WT, Angus DC (2004) Severe sepsis epidemiology: sampling, selection, and society. Crit Care 8:222-226

5. Alberti C, Brun-Buisson C, Burchardi H, Martin C, Goodman S, Artigas A et al (2002) Epidemiology of sepsis and infection in ICU patients from an international multicentre cohort study. Intensive Care Med 28:108-121

6. Hotchkiss RS, Karl IE (2003) The pathophysiology and treatment of sepsis. N Engl J Med 348:138-150

7. Mikkelsen ME, Miltiades AN, Gaieski DF, Goyal M, Fuchs BD, Shah CV et al (2009) Serum lactate is associated with mortality in severe sepsis independent of organ failure and shock. Crit Care Med 37:1670-1677

8. Filho RR, Rocha LL, Corrêa TD, Pessoa CM, Colombo G, Assuncao MS (2016) Blood lactate levels cutoff and mortality prediction in sepsis-time for a reappraisal? a retrospective cohort study. Shock 46:480-485

9. Schuetz P, Birkhahn R, Sherwin R, Jones AE, Singer A, Kline JA et al (2017) Serial procalcitonin predicts mortality in severe sepsis patients: results from the multicenter procalcitonin MOnitoring SEpsis (MOSES) study. Crit Care Med 45:781-789

10. Song J, Park DW, Moon S, Cho HJ, Park JH, Seok H et al (2019) Diagnostic and prognostic value of interleukin-6, pentraxin 3 , and procalcitonin levels among sepsis and septic shock patients: a prospective controlled study according to the Sepsis-3 definitions. BMC Infect Dis 19:968

11. Narasimha A, Harendra Kumar ML (2011) Significance of Hematological Scoring System (HSS) in early diagnosis of neonatal sepsis. Indian J Hematol Blood Transfus 27:14-17

12. Kaushik R, Gupta M, Sharma M, Jash D, Jain N, Sinha N et al (2018) Diagnostic and prognostic role of Neutrophil-to-Lymphocyte Ratio in early and late phase of sepsis. Indian J Crit Care Med 22:660-663

13. Matsubara T, Yamakawa K, Umemura Y, Gando S, Ogura H, Shiraishi A et al (2019) Significance of plasma fibrinogen level and antithrombin activity in sepsis: a multicenter cohort study using a cubic spline model. Thromb Res 181:17-23

14. Mitra P, Guha D, Nag SS, Mondal BC, Dasgupta S (2017) Role of plasma fibrinogen in diagnosis and prediction of short term outcome in neonatal sepsis. Indian $\mathbf{J}$ Hematol Blood Transfus 33:195-199

15. Vardon-Bounes F, Ruiz S, Gratacap MP, Garcia C, Payrastre B, Minville V (2019) Platelets are critical key players in sepsis. Int J Mol Sci 20:3494

16. Jackson SR, Carter JM (1993) Platelet volume: laboratory measurement and clinical application. Blood Rev 7:104-113

17. Gao Y, Li Y, Yu X, Guo S, Ji X, Sun T et al (2014) The impact of various platelet indices as prognostic markers of septic shock. PLoS ONE 9:e103761

18. Zampieri FG, Ranzani OT, Sabatoski V, de Souza HP, Barbeiro $\mathrm{H}$, da Neto LM et al (2014) An increase in mean platelet volume after admission is associated with higher mortality in critically ill patients. Ann Intensive Care 4:20

19. Guclu E, Durmaz Y, Karabay O (2013) Effect of severe sepsis on platelet count and their indices. Afr Health Sci 13:333-338

20. Becchi C, Al Malyan M, Fabbri LP, Marsili M, Boddi V, Boncinelli S (2006) Mean platelet volume trend in sepsis: is it a useful parameter? Minerva Anestesiol 72:749-756

21. Woth G, Varga A, Ghosh S, Krupp M, Kiss T, Bogár L et al (2011) Platelet aggregation in severe sepsis. J Thromb Thrombolysis 31:6-12

22. Assinger A, Schrottmaier WC, Salzmann M, Rayes J (2019) Platelets in sepsis: an update on experimental models and clinical data. Front Immunol 10:1687

23. Vincent JL, Yagushi A, Pradier O (2002) Platelet function in sepsis. Crit Care Med 30:S313-S317

24. Van der Lelie J, Von dem Borne AK (1983) Increased mean platelet volume in septicaemia. J Clin Pathol 36:693-696

25. Robbins G, Barnard DL (1983) Mean platelet volume changes in infection. J Clin Pathol 36:1320

26. Orak M, Karakoç Y, Ustundag M, Yildirim Y, Celen MK, Güloglu C (2018) An investigation of the effects of the mean platelet volume, platelet distribution width, platelet/lymphocyte ratio, and platelet counts on mortality in patents with sepsis who applied to the emergency department. Niger J Clin Pract 21:667-671

27. Akarsu S, Taskin E, Kilic M, Ozdiller S, Gurgoze MK, Yilmaz E et al (2005) The effects of different infectious organisms on platelet counts and platelet indices in neonates with sepsis: is there an organism-specific response? J Trop Pediatr 51:388-391

28. Venkata C, Kashyap R, Farmer JC, Afessa B (2013) Thrombocytopenia in adult patients with sepsis: incidence, risk factors, and its association with clinical outcome. J Intensive Care 1:9

29. Vincent JL, Castro P, Hunt BJ, Jörres A, Praga M, Rojas-Suarez J et al (2018) Thrombocytopenia in the ICU: disseminated intravascular coagulation and thrombotic microangiopathies-what intensivists need to know. Crit Care 22:158

30. Gregoriano C, Heilmann E, Molitor A, Schuetz P (2020) Role of procalcitonin use in the management of sepsis. $\mathbf{J}$ Thorac Dis 12:S5-S15

31. Demirdal T, Sen P, Nemli SA (2018) Diagnostic value of procalcitonin in predicting bacteremia in intensive care unit. Indian $\mathrm{J}$ Crit Care Med 22:78-84

32. Kim CH, Kim SJ, Lee MJ, Kwon YE, Kim YL, Park KS et al (2015) An increase in mean platelet volume from baseline is associated with mortality in patients with severe sepsis or septic shock. PLoS ONE 10:e0119437

33. Aydemir H, Piskin N, Akduman D, Kokturk F, Aktas E (2015) Platelet and mean platelet volume kinetics in adult patients with sepsis. Platelets 26:331-335

Publisher's Note Springer Nature remains neutral with regard to jurisdictional claims in published maps and institutional affiliations. 